\title{
Analyzing Nonlinear System Stability of a New Hydraulic Bilateral Rolling Shear
}

\author{
HeYong HAN, ${ }^{1)}$ HongZhou $\mathrm{LI}^{2)}{ }^{2)} \mathrm{Jia} \mathrm{LI}^{1{ }^{1} *}{ }^{*} \mathrm{KeJun} \mathrm{MIAO},{ }^{1)}$ Jing WANG ${ }^{1)}$ and CongMei WEI ${ }^{3)}$ \\ 1) Heavy Machinery Eng. Research Center of Education Ministry, Taiyuan University of Science and Technology, Taiyuan, \\ 030024 China. $\quad 2)$ Hebei Wenfeng Iron and Steel Co. Ltd, Hebei, Wuan, 056300 PR China. \\ 3) School of Mechanical Engineering of Taiyuan University of Science and Technology, Taiyuan, 030024 China.
}

(Received on March 17, 2016; accepted on June 21, 2016; J-STAGE Advance published date: September $17,2016)$

\begin{abstract}
New hydraulic bilateral rolling shear is a composite connecting rod shearing mechanism with the upper blade driven by servo-cylinders. This new creative system would be unstable because of interaction between the mechanical dynamics and hydraulic dynamics of the machine. What's more, the positive feedback between hydro-cylinder \& mechanic, which produces instability phenomenon. In this model, all instabilities result from the control of the fuel. In this study, by using the unsymmetrical valve to control unsymmetrical cylinder, this hydraulic servo model could get the nonlinear equation of the state between force and cylinder flow. Lyapunov stability theory is also used to analyze the stability of system to prove the consequence. According to the recorded data, actual trajectory agree well with given trajectory, which show that method in this paper can meet the practical demand in engineering. In addition, the experimental results show that the nonlinear system with multiple degrees of freedom is stable and its performance is outstanding.
\end{abstract}

KEY WORDS: stability analysis; new hydraulic bilateral rolling shear; lyapunov; nonlinear system.

\section{Introduction}

The bilateral rolling shears is one of the most important device to cut the width of a medium steel plate, it could cut both sides of the plate simultaneously via the stepping cut method, ${ }^{1,2)}$ as shown in Fig. 1. The shearing mechanism of the new hydraulic bilateral rolling shear is driven by servo cylinders, which are positioned horizontally on both sides of the frame to drive the arcuate upper blade of the longitudinal

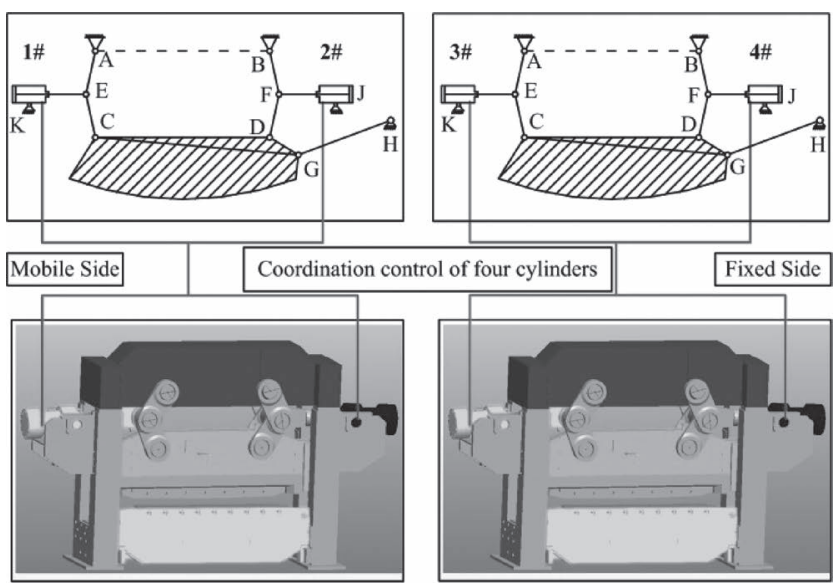

Fig. 1. New hydraulic bilateral rolling shear.

* Corresponding author: E-mail: mkj_lj@126.com

DOI: http://dx.doi.org/10.2355/isijinternational.ISIJINT-2016-152 shearing plate. Generally, the trajectory of upper blade is complex and the four cylinders' displacements are interacting, the opening of the servo valve is approximately $70 \%$ to $80 \%$, which is a typical nonlinear system with multiple DOF. By using the unsymmetrical valve controlling unsymmetrical cylinder, the pressure and the flow strike caused by the cylinder commutation can be decreased. ${ }^{3,4)}$ The shearing mechanism linked to the arcuate upper blade driven by four servo cylinders exhibiting a rolling cut motion. Therefore, the stability of the valve that controlled the cylinder hydraulic system determined the performance of the hydraulic bilateral rolling shear. At present, a prototype of the device developed and applied in a production test. While the device has a simple structure, can nevertheless has a large shearing force, and high control accuracy. Therefore, the stability \& reliability of this shearing mechanism is so important that it might be necessary for us to consider more about it.

Moreover, complicated nonlinear system such as the new hydraulic bilateral rolling shear, which can hardly realize cylinders coupled control and damping control, based on unsymmetrical actuators had poor adaptability and instability. In this type of system, the hydro-mechanical interaction had a position feedback, which can create instability. Numerous scholars have studied the stability of mechanical structure. Interactive dynamics of a backhoe was researched by Margolis D and Shim T based on Bonds graphs. ${ }^{5)}$ Gentner, Ch studied the instability phenomena in pump turbines by experimental and numerical analysis. ${ }^{6)}$ Li J studied system stability of multi-DOF multilateral 
haptic systems by the Llewellyn criterion, which confirmed the theoretical stability conditions. ${ }^{7)}$ Stability of uncertain MIMO linear systems with multiple time-delays and external disturbances was analyzed by Vahid J. M. based on Lyapunov-Krasovskii functional and the conditions for stabilization were obtained. ${ }^{8)}$ Among the analyses of the stability of multi-DOF hydraulic systems, few studies conducted on multi-cylinder and single-cylinder systems. In a new hydraulic bilateral rolling shear, which is a nonlinear system controlled by four coupled cylinders, the lack of coordination among the control cylinders can eventually lead to system instability and structural damage.

By utilizing the unsymmetrical valve to control the unsymmetrical cylinder, we got the nonlinear equation between the pressure and the flow of the hydraulic servo cylinders. Thus, the stability of this nonlinear system is analyzed by Lyapunov Stability Theory. What's more, the comparison between the given displacements of the four cylinders \& the actual displacements also prove the accuracy of the equation we got.

\section{Building the Mathematical Model of the Cylinder Controlled by the Valve of the Hydraulic Servo System}

For this mechanism, the cylinder controlled by the valve, of the servo system equipped with new hydraulic bilateral rolling shear, the valve orifice area and the cylinder are unsymmetrical. Besides this, the opening of the servo valve has a large stroke and a large variable load. So it is unsuitable for evaluating the stability of the system using the classical linear system model. Thus, establishing the mathematical model using the unsymmetrical cylinder controlled by the unsymmetrical valve ${ }^{9,10)}$ is necessary.

\subsection{Developing the Throttle Formulas of Each Win- dow of the Unsymmetrical Servo Valve}

This study mainly focuses on the input and output areas through the throttle windows of the unsymmetrical valve, which exhibit the following proportion:

$$
w_{2} / w_{1}=n
$$

Where $w_{1}$ is the area gradients of windows 1,$4 ; w_{2}$ is the area gradients of windows 2, 3; and $\mathrm{n}$ is a constant that is smaller than 1 .

When $\mathrm{n}<1$, the valve is unsymmetrical; when $\mathrm{n}=1$, the valve is symmetrical.

For the unsymmetrical hydraulic cylinder,

$$
A_{2} / A_{1}=m
$$

Where $A_{1}$ is the effective action area of the rear cavity of the cylinder, and $A_{2}$ is the effective action area of its rod cavity.

As shown in Fig. 2, the throttle formulas of valve ports $1,2,3$, and 4 are respectively as follows:

$$
\begin{aligned}
& q_{s v 1}=\left\{\begin{array}{cc}
0 & x_{v} \leq \Delta_{1} \\
c_{d} w_{1}\left(x_{v}-\Delta_{1}\right) \sqrt{2\left(p_{s}-p_{1}\right) / \rho} & x_{v}>\Delta_{1}
\end{array}\right. \\
& q_{s v 2}=\left\{\begin{array}{cc}
0 & x_{v} \geq \Delta_{2} \\
-c_{d} w_{2}\left(x_{v}+\Delta_{2}\right) \sqrt{2\left(p_{s}-p_{2}\right) / \rho} & x_{v}<\Delta_{2}
\end{array}\right.
\end{aligned}
$$

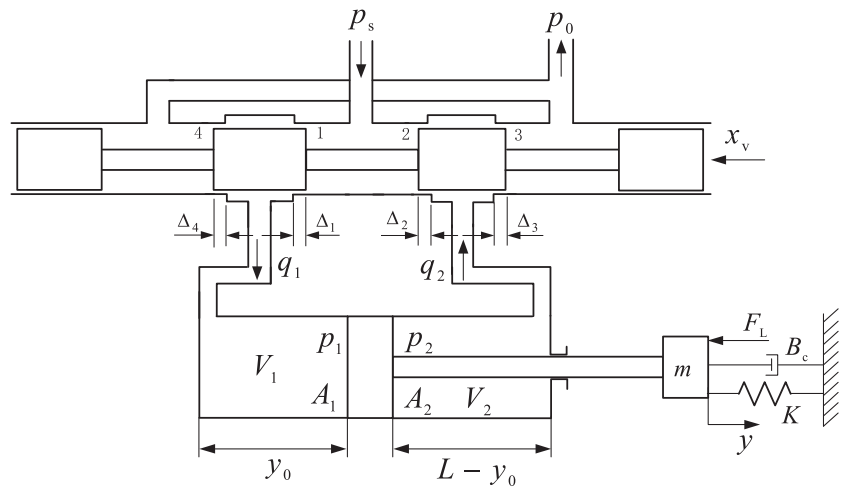

Fig. 2. Principle of the unsymmetrical cylinder controlled by the servo valve.

$$
\begin{gathered}
q_{s v 3}=\left\{\begin{array}{cc}
0 & x_{v} \leq \Delta_{3} \\
c_{d} w_{3}\left(x_{v}-\Delta_{3}\right) \sqrt{2\left(p_{2}-p_{0}\right) / \rho} & x_{v}>\Delta_{3}
\end{array}\right. \\
q_{s v 4}=\left\{\begin{array}{cc}
0 & x_{v} \leq \Delta_{4} \\
-c_{d} w_{4}\left(x_{v}+\Delta_{4}\right) \sqrt{2\left(p_{1}-p_{0}\right) / \rho} & x_{v}>\Delta_{4}
\end{array}\right.
\end{gathered}
$$

Where $C_{\mathrm{d}}$ is the flow coefficient; $x_{v}$ is the displacement of the valve core; $\Delta_{\mathrm{i}}$ are the openings of the windows of the valve cores $(i=1 \sim 4) ; p_{\mathrm{s}}$ is the supply pressure $(\mathrm{Pa}) ; \rho$ is the oil density $\left(\mathrm{kg} / \mathrm{m}^{3}\right) ; q_{\mathrm{sv} 1}, q_{\mathrm{sv} 2}, q_{\mathrm{sv} 3}, q_{\mathrm{sv} 4}$ are the flow of windows of the valve cores $\left(\mathrm{m}^{3} / \mathrm{s}\right)$.

The sliding displacement moves forward, as shown in Fig. 2. The flow-in and flow-out of the cylinders are respectively as follows.

$$
\begin{aligned}
& Q_{1}=f_{1}\left(x_{v}, p_{1}\right)=q_{s v 1}-q_{s v 4} \\
& Q_{2}=f_{2}\left(x_{v}, p_{2}\right)=q_{s v 3}-q_{s v 2}
\end{aligned}
$$

Assuming that $\left|\Delta_{1}\right|=\left|\Delta_{2}\right|=\left|\Delta_{3}\right|=\left|\Delta_{4}\right|, \quad \Delta_{1}=-\Delta_{4}$, $\Delta_{3}=-\Delta_{2}$, then the flow of the rear cavity is as follows.

$$
\begin{aligned}
& Q_{1}=c_{d} w_{1}\left(x_{v}-\Delta_{1}\right) \sqrt{2\left(p_{s}-p_{1}\right) / \rho} x_{v}>\Delta_{1} \ldots \ldots \\
& Q_{1}^{\prime}=c_{d} w_{4}\left(x_{v}+\Delta_{4}\right) \sqrt{2\left(p_{1}-p_{0}\right) / \rho} x_{v} \leq \Delta_{4} \ldots \ldots
\end{aligned}
$$

Meanwhile, the flow of the rod cavity is as follows:

$$
\begin{aligned}
& Q_{2}=c_{d} w_{3}\left(x_{v}-\Delta_{3}\right) \sqrt{2\left(p_{2}-p_{0}\right) / \rho} x_{v}>\Delta_{3} \ldots . \\
& Q_{2}^{\prime}=c_{d} w_{4}\left(x_{v}+\Delta_{2}\right) \sqrt{2\left(p_{\mathrm{s}}-p_{2}\right) / \rho} x_{v} \leq \Delta_{2} \ldots \ldots
\end{aligned}
$$

Where, $\mathrm{Q}_{1}, \mathrm{Q}_{1}{ }^{\prime}$ are the flow of the rear cavity that is moving forward and backward.

$\mathrm{Q}_{2}, \mathrm{Q}_{2}{ }^{\prime}$ are the flow of the rod cavity that is moving forward and backward.

When $\mathrm{m}=\mathrm{n}$, the backward impact can be reduced and the system achieves a wide range of load variations. In addition, an unsymmetrical valve control completely matches an unsymmetrical cylinder, which can eliminate the adverse effect of the unsymmetrical cylinder on the pressure characteristics of the system. ${ }^{4)}$

\subsection{Pressure and Flow Characteristics of the Cylinder under a Steady State}

$$
\frac{Q_{2}}{Q_{1}}=\frac{c_{d} w_{3}\left(x_{v}-\Delta_{3}\right)}{c_{d} w_{1}\left(x_{v}-\Delta_{1}\right)} \sqrt{\frac{p_{2}-p_{0}}{p_{s}-p_{1}}}
$$


From Eq. (2-9), we obtain,

$$
p_{s}=p_{1}+p_{2}
$$

When the cylinder is stable, it satisfies the following equilibrium equation:

$$
p_{1} A_{1}-P_{2} A_{2}=P_{L} A_{1}
$$

$P_{\mathrm{L}}$ is given by

$$
p_{L}=p_{1}-m p_{2}
$$

The output power of the hydraulic cylinder is as follows:

$$
N=P_{1} Q_{1}-P_{2} Q_{2}=\left(P_{1}-m P_{2}\right) Q_{1}=Q_{L} P_{L}
$$

Then, load flow is given by

$$
Q_{L}=Q_{1}
$$

Thus, the load flow of the sliding valve is equated to the flow of the rear cavity of the hydraulic cylinder, which is connected to the valve port.

Based on Eqs. (2-9), (2-10), and (2-11), the load flows can be expressed as follows:

$$
\begin{gathered}
Q_{L+}=c_{d} w_{1}\left(x_{v}-\Delta_{1}\right) \sqrt{\frac{2\left(p_{s}-p_{L}\right)}{\rho(1+m)}} \quad x_{v}>\Delta_{1} \ldots \\
Q_{L-}=c_{d} w_{4}\left(x_{v}-\Delta_{1}\right) \sqrt{\frac{2\left(m p_{s}+p_{L}\right)}{\rho(1+m)}} x_{v} \leq \Delta_{4} \cdots
\end{gathered}
$$

2.3. Basic Equation of the Asymmetric Servo Valve Control by the Asymmetrical Cylinder

When the cylinder moves to the right, that is, $\mathrm{x}_{\mathrm{v}}>\Delta_{1}$, we obtain the following equation: ${ }^{11)}$

$$
Q_{L}=c_{d} w_{1}\left(x_{v}-\Delta_{1}\right) \sqrt{\frac{2\left(p_{s}-p_{L}\right)}{\rho(1+m)}} x_{v}>\Delta_{1}
$$

Where, $P_{L}$ is the pressure of the sliding valve.

Considering the internal and external leakage of the cylinders and the compressibility of oil, the flow continuity equation of the hydraulic cylinder rear cavity is as follows:

$$
Q_{1}=\frac{d V_{1}}{d_{t}}+c_{i p}\left(p_{1}-p_{2}\right)+\frac{V_{1}}{\beta_{e}} \frac{d p_{1}}{d_{t}}
$$

Where $V_{1}=V_{\mathrm{d} 1}+A_{1}\left(y_{0}+y\right), V_{2}=V_{\mathrm{d} 2}+A_{2}\left(L-L_{0}-y\right)$.

$V_{\mathrm{d} 1}, V_{\mathrm{d} 2}$ denote the dead space volumes of the two cavities of the cylinder, which comprises the pipeline volume from the valve to the cylinder.

$y$ is the displacement of the piston, $c_{\text {ip }}$ is the internal leakage of cylinder, and $c_{\text {ep }}$ is the external leakage of cylinder.

$A_{1}$ is the effective action area of the rear cavity of the cylinder.

$A_{2}$ is the effective action area of the rod cavity of the cylinder.

Based on Eqs. (2-10), (2-11), and (2-15), we obtain the following:

$$
Q_{L}=A_{1} \dot{y}+c_{t p} p_{L}+c_{i p} \mu p_{s}+\frac{V_{t}}{4 \beta_{e}} \frac{d p_{L}}{d t}
$$

Where $c_{\mathrm{tp}}=2 c_{\mathrm{ip}} / 1+m, \mu=m-1 / m+1, V_{1 \min }=V_{\mathrm{d} 1}+A_{1} y_{0}$, $V_{1 \max }=V_{\mathrm{d} 1}+A_{1} L$, and $L$ is the length of the hydraulic cylinder. In general, $V_{1}=\left(V_{1 \min }+V_{1 \max }\right) / 2$ and equivalent volume $V_{\mathrm{t}}=4 V_{1} / 1+m=4 V_{\mathrm{d} 1}+2 A_{1}\left(y_{0}+L\right) / 1+m$.
Based on the present analysis of the stability of the mechanism of the cylinder controlled by the valve, it is not difficult to find that when the piston locates at the medium position, the system reflects the poorest stability. Thus, the value of $V_{\mathrm{t}}$ should remain in the piston position, i.e., $V_{\mathrm{t}}=$ $A_{1} L / 3$.

The equilibrium equation of the piston is as follows:

$$
A_{1} P_{1}-A_{2} P_{2}=A_{1} p_{L}=m_{t} \ddot{y}+B \dot{y}+k y+F .
$$

Where $m_{\mathrm{t}}$ is the mass piston and the load converted into the piston, $B$ is the viscous damping coefficient of the piston and the loads, $k$ is spring stiffness of the loads, and $F$ is the loads.

\section{Evaluating the Stability of the Nonlinear System}

Based on Eqs. (2-14), (2-16), and (2-17), the nonlinear state equations can be linearized as follows:

$$
\begin{aligned}
& x=\left[\begin{array}{lll}
x_{1} & x_{2} & x_{3}
\end{array}\right]^{\mathrm{T}}=\left[\begin{array}{lll}
y & \dot{y} & P_{L}
\end{array}\right]^{\mathrm{T}} \\
& \dot{x}_{1}=x_{2} \\
& \dot{x}_{2}=\frac{1}{m_{t}}\left(-k x_{1}-b x_{2}-F+A_{1} x_{3}\right) \\
& \dot{x}_{3}=\frac{4 \beta_{e}}{V_{t}}\left(Q_{L}-A_{1} x_{2}-c_{t p} x_{3}-\mu c_{i p} p_{s}\right)
\end{aligned}
$$

The preceding formula is terminated as follows:

$$
\left[\begin{array}{c}
\dot{x}_{1} \\
\dot{x}_{2} \\
\dot{x}_{3}
\end{array}\right]=\left[\begin{array}{ccc}
0 & 1 & 0 \\
-\frac{k}{m_{t}} & -\frac{B}{m_{t}} & \frac{A_{1}}{m_{t}} \\
0 & -\frac{4 \beta_{e} A_{1}}{V_{t}} & \frac{4 \beta_{e}}{V_{t}}\left(\frac{Q_{L}}{x_{3}}-c_{t p}\right)
\end{array}\right]\left[\begin{array}{c}
x_{1} \\
x_{2} \\
x_{3}
\end{array}\right]+\left[\begin{array}{c}
0 \\
-\frac{F}{m_{t}} \\
4 \beta_{e} \mu c_{i p} p_{s} \\
V_{t}
\end{array}\right]
$$

The state variable of the servo valve control system is converted into state vector as shown in Eq. (3-2).

$$
\begin{gathered}
\dot{X}_{1}=\bar{A}_{1} X_{1}+D_{1} \\
Y_{1}=C_{1} X_{1}
\end{gathered}
$$

As shown in Eq. (3-2),

$$
\begin{gathered}
\dot{X}_{1}=\left[\begin{array}{ccc}
\dot{x}_{1} & \dot{x}_{2} & \dot{x}_{3}
\end{array}\right]^{\mathrm{T}} ; \\
X_{1}=\left[\begin{array}{lll}
x_{1} & x_{2} & x_{3}
\end{array}\right]^{\mathrm{T}} ; \\
\bar{A}_{1}=\left[\begin{array}{ccc}
0 & 1 & 0 \\
-\frac{k}{m_{t}} & -\frac{B}{m_{t}} & \frac{A_{1}}{m_{t}} \\
0 & -\frac{4 \beta_{e} A_{1}}{V_{t}} & \frac{4 \beta_{e}}{V_{t}}\left(\frac{Q_{L}}{x_{3}}-c_{t p}\right)
\end{array}\right] ; \\
D_{1}=\left[\begin{array}{ccc}
0 & -\frac{F}{m_{t}} & -\frac{4 \beta_{e} \mu c_{i p} p_{s}}{V_{t}}
\end{array}\right]^{\mathrm{T}} ;
\end{gathered}
$$




$$
C_{1}=E \text {. }
$$

\subsection{Analyzing the Stability of Four Cylinders Con- trolled by the Servo Valve}

The new hydraulic bilateral rolling shear is a nonlinear system with multiple DOF. This system consists of four hydraulic servo cylinders driving upper blade motion. The load flow equation, continuity equation, and force equilibrium equation of each hydraulic servo cylinder must be independent; thus, the method for establishing a state equation with multiple DOF based on the differential equation is the same as that for establishing a state equation of a single hydraulic cylinder.

$$
\begin{gathered}
\dot{X}_{(12 \times 1)}=\bar{A}_{(12 \times 12)} X_{(12 \times 1)}+D_{(12 \times 1)} \\
Y_{(4 \times 1)}=C_{(4 \times 12)} X_{(12 \times 1)} \ldots \ldots \ldots \ldots
\end{gathered}
$$

As shown in Eqs. (3-3) and (3-4),

$$
\begin{gathered}
\bar{A}=\operatorname{diag}\left\{\bar{A}_{1}, \bar{A}_{2}, \bar{A}_{3}, \bar{A}_{4}\right\} \\
D=\operatorname{diag}\left\{D_{1}, D_{2}, D_{3}, D_{4}\right\} \\
C=\operatorname{diag}\left\{C_{1}, C_{2}, C_{3}, C_{4}\right\} \\
\dot{X}=\left[\begin{array}{llll}
\dot{X}_{1} & \dot{X}_{2} & \dot{X}_{3} & \dot{X}_{4}
\end{array}\right]^{\mathrm{T}} \\
Y=\left[\begin{array}{llll}
Y_{1} & Y_{2} & Y_{3} & Y_{4}
\end{array}\right]^{\mathrm{T}}
\end{gathered}
$$

The two preceding equations were transformed into matrix form, as shown in Eqs. (3-5) and (3-6).

$$
\left[\begin{array}{l}
\dot{X}_{1} \\
\dot{X}_{2} \\
\dot{X}_{3} \\
\dot{X}_{4}
\end{array}\right]=\left[\begin{array}{cccc}
\bar{A}_{1} & 0 & 0 & 0 \\
0 & \bar{A}_{2} & 0 & 0 \\
0 & 0 & \bar{A}_{3} & 0 \\
0 & 0 & 0 & \bar{A}_{4}
\end{array}\right]\left[\begin{array}{l}
X_{1} \\
X_{2} \\
X_{3} \\
X_{4}
\end{array}\right]+\left[\begin{array}{cccc}
D_{1} & 0 & 0 & 0 \\
0 & D_{2} & 0 & 0 \\
0 & 0 & D_{3} & 0 \\
0 & 0 & 0 & D_{4}
\end{array}\right]
$$

$$
\left[\begin{array}{l}
Y_{1} \\
Y_{2} \\
Y_{3} \\
Y_{4}
\end{array}\right]=\left[\begin{array}{cccc}
C_{1} & 0 & 0 & 0 \\
0 & C_{2} & 0 & 0 \\
0 & 0 & C_{3} & 0 \\
0 & 0 & 0 & C_{4}
\end{array}\right]\left[\begin{array}{l}
X_{1} \\
X_{2} \\
X_{3} \\
X_{4}
\end{array}\right]
$$

The state equation of the shear mechanism is a 4D matrix equation whose stability is analyzed by the first Lyapunov method.

For $\dot{x}=A x$, if the eigenvalues of $A$ possess a negative real part, then the system is asymptotically stable according to the first Lyapunov method.

According to Eq. (3-5), the eigenvalues of matrix $\bar{A}$ are $\left|\bar{A}_{1}\right|,\left|\bar{A}_{2}\right|,\left|\bar{A}_{3}\right|$, and $\left|\bar{A}_{4}\right|$. The systems of the four hydraulic servo cylinders must be independent to analyze the eigenvalues of $A$ as follows:

$$
\begin{aligned}
\left|\bar{A}_{1}\right| & =-\frac{4 k \beta_{e}}{m_{t} V_{t}}\left(\frac{Q_{L}}{x_{3}}-c_{t p}\right) \\
& =-\frac{4 k \beta_{e}}{m_{t} V_{t}}\left(c_{d} w\left(x_{v}-\Delta_{1}\right) \sqrt{\frac{2\left(P_{s}-P_{L}\right)}{\rho P_{L}^{2}}}\right)
\end{aligned}
$$

The parameters of the hydraulic system and the load summary are presented in Table $\mathbf{1}$.

Based on the analysis and computation results, $\left|\bar{A}_{1}\right|<0$, and thus, $\left|\bar{A}_{2}\right|<0,\left|\bar{A}_{3}\right|<0$, and $\left|\bar{A}_{4}\right|<0$. The eigenvalues of matrix $\bar{A}$ are less than 0 ; hence, the nonlinear system with multiple DOF is stable. The stability of a single cylinder controlled by the servo valve should also be satisfied to ensure the normal operation of the shear mechanism of the bilateral rolling shear. The stability analysis of a singlecylinder system is as follows.

\subsection{Analyzing the Stability of a Single-cylinder Con- trolled by the Servo Valve}

This study analyzes the stability of the extending servo cylinder. The speed and acceleration of the cylinder are positive. Based on the second Lyapunov method and according to the conservation of oil energy, the Lyapunov function is developed. Assume that $V(x, t)$ is Lee's function and the state equation of the system is $\dot{x}=f(x, t)$, whose equilibrium state is $f(0, t)=0$. The energy stored by the physical system is positive definite, and thus, $V(x, t)>0$.

The Lyapunov function $V(x)$ is established as follows:

$V=p_{1} V_{1}-\frac{1}{2} \rho V_{1} \dot{y}^{2}-p_{2} V_{2}-\frac{1}{2} \rho V_{2} \dot{y}^{2}-\frac{1}{2} m_{t} \dot{y}^{2}-\frac{1}{2} k y^{2} \ldots$

The first-order derivative of $V(x)$ is given by

$$
\begin{aligned}
\dot{V} & =p_{1} A_{1} \dot{y}-\frac{1}{2} \rho A_{1}\left(\dot{y}^{3}+2 y \ddot{y} \ddot{y}\right)-\frac{1}{2} \rho A_{2}\left(\dot{y}^{3}+2 y \ddot{y} \ddot{y}\right) \\
& -p_{2} A_{2} \dot{y}-m \ddot{y} \ddot{y}-k y \dot{y} \\
\dot{V} & =\left(p_{1} A_{1} \dot{y}-\rho A_{1} y \dddot{y} \ddot{y}\right)-\frac{1}{2} \rho A_{1} \dot{y}^{3}-p_{2} A_{2} \dot{y}-\frac{1}{2} \rho A_{2} \dot{y}^{3} \\
& -\rho A_{2} y \dddot{y} \ddot{y}-m_{t} \ddot{y} \ddot{y}-k y \dot{y}
\end{aligned}
$$

where $F_{1}$ is the force of the rear cavity, $F_{2}$ is the force of the rod cavity, and $\rho A_{1} \ddot{y}=F_{1}-F_{2}-F_{L}$.

$$
\begin{aligned}
\dot{V} & =\dot{y}\left(p_{1} A_{1}-F_{1}\right)+\dot{y}\left(F_{2}-p_{2} A_{2}\right)+\dot{y}\left(F_{L}-k y\right) \\
& -m_{t} \ddot{y} \ddot{y}-\frac{1}{2} \rho A_{1} \dot{y}^{3}-\frac{1}{2} \rho A_{2} \dot{y}^{3}-\rho A_{2} y \ddot{y} \ddot{y}
\end{aligned}
$$

(a) Given that $\dot{V}$ is negative definite, the first three $\dot{V}$ are satisfied as follows:

$$
\dot{y}\left(p_{1} A_{1}-F_{1}\right)+\dot{y}\left(F_{2}-p_{2} A_{2}\right)+\dot{y}\left(F_{L}-k y\right)<0 .
$$

Table 1. Parameters of the hydraulic cylinder system.

\begin{tabular}{lcc}
\hline \multicolumn{1}{c}{ Content } & $\begin{array}{c}\text { Quantity } \\
\text { symbols }\end{array}$ & $\begin{array}{c}\text { Parameter } \\
\text { values }\end{array}$ \\
\hline Valve flow coefficient & $c_{\mathrm{d}}$ & 0.6 \\
Grads of valve window & $w$ & $16 \pi \mathrm{mm}$ \\
Viscous damping coefficient & $\beta_{\mathrm{e}}$ & $750 \mathrm{MPa}$ \\
Oil density & $\rho$ & $900 \mathrm{~kg} / \mathrm{m} 3$ \\
Supply pressure & $P_{s}$ & $28 \mathrm{MPa}$ \\
Mass of hydraulic cylinder piston rod & $m_{\mathrm{t}}$ & $950 \mathrm{~kg}$ \\
Ratio of effective area & $m$ & 0.5 \\
Elastic stiffness of load & $k$ & $107 \mathrm{~N} / \mathrm{mm}$ \\
\hline
\end{tabular}


Where $p_{1} A_{1}-F_{1}=0, F_{2}-p_{2} A_{2}=0$, and $\dot{y}\left(F_{L}-k y\right)<0$, $\dot{y}>0$; thus, $F_{L}-k y<0$ ( $k$ is the elastic coefficient with a value of $20 \times 10^{4} \mathrm{~N} / \mathrm{mm}$ ). In view of occasional tear phenomenon of the sheared steel plate in the process of shearing are discussed in this paper. It has been stated ${ }^{12)}$ that the maximum shear load measured during actual production is $5.12 \times 10^{6}$ $\mathrm{N} / \mathrm{mm}$; hence, $\mathrm{y}>26 \mathrm{~mm}$ is required. When the displacement of the hydraulic cylinder is $26 \mathrm{~mm}$, the maximal descending displacement of the upper blade is approximately $1 \mathrm{~mm}$. Based on the practical production requirements, the distance between the upper blade and the steel plate is approximately $80 \mathrm{~mm}$, i.e., when the descending displacement of the upper blade is $80 \mathrm{~mm}$, the upper blade begins to shear the plate. In other words, as long as the shearing mechanism is designed, the position of hydraulic cylinder is greater than $F_{\mathrm{L}} / k$, the stability of the hydraulic system can be satisfied. The first three calculated value are obviously less than zero. Moreover, works for null force can also satisfy the stability condition. The above research will provide the references for stability study of valve control cylinder system. By collecting pressure signals of pressure sensor installed on the hydraulic system, a big change in pressure fluctuations will appear, so the effect of pressure changes on system stability can not be accurately analyzed. In addition, in the design of the actual device, system actual set pressure is higher than actual working pressure, and system pressure stability is the prerequisite for normal operation of equipment. Therefore, effect on the stability of the system pressure on the system is less analyzed in this paper.

(b) For $-m_{t} \ddot{y} \ddot{y}-\frac{1}{2} \rho A_{1} \dot{y}^{3}-\frac{1}{2} \rho A_{2} \dot{y}^{3}-\rho A_{2} y \ddot{y} \ddot{y}$, each item is less than zero and satisfies the negative definite condition, which in turn, satisfies the stable condition of the second Lyapunov method. Therefore, using the unsymmetrical valve to control the unsymmetrical cylinder can achieve stability.

\section{Experiment Research}

\subsection{Experimental Procedures}

The new hydraulic bilateral rolling shear was designed by the Taiyuan University of Science and Technology, and produced by the Taiyuan Heavy Machinery Group. A given numerical of the theoretical calculation need to be verified through the experiment. The cylinder displacement is detected by the displacement sensor, which are both installed on the servo cylinder. The displacement of the upper blade cannot be directly measured, and thus, the displacement data of the cylinder are gathered. The stability of the upper blade is determined by stability of the hydraulic cylinder, so that positional deviation of the upper blade can be replaced by positional deviation of cylinders. To resist disturbance of external load and ensure the robustness of the hydraulic system, according to selection manual RE 30139/05.07, VT-HNC-100 controller of Rexroth is applied. So the displacement of hydraulic cylinders is controlled under the outside interference in real-time, which lead to that displacement error is reduced and robustness of the system is improved. The hydraulic servo system adopts a 4WRTE servo valve produced by Bosch Rexroth (Lohr am Main, Germany). The gradient ratio of the valve orifice area is 0.5 and the effective area ratio between the two cavities in the servo hydraulic cylinder is 0.5 based on the bearing capacity of the servo valve.

\subsection{Experimental Results and Discussion}

The error between the actual and given displacements is analyzed by collecting the actual displacements of the four hydraulic servo cylinders and the stability and reliability of the shear mechanism are validated. ${ }^{13)}$ Comparing the actual and given displacements in the field as shown in Fig. 3, the four cylinders of the bilateral rolling shear are determined to be stable. As shown in Fig. 3(a), the error of 1 \# cylinder between the actual and given displacements is $0.55 \%$ to $15.3 \%$, that of $2 \#$ cylinder is $0.08 \%$ to $13.3 \%$ (Fig. 3(b)), that of $3 \#$ cylinder is $0.1 \%$ to $11.3 \%$ (Fig. 3(c)), and that of $4 \#$ cylinder is $0.4 \%$ to $5.8 \%$ (Fig. $3(\mathrm{~d})$ ). Reason of position deviation increased mainly has the following points: 1 . Acceleration is relatively large when hydraulic cylinder is started, a larger flow is needed to meet the position requirements and the servo valve spool opening is relatively large, the response frequency of the servo valve is reduced, which resulting in the displacement error of hydraulic cylinder is increased. 2. With the increase of shear steel plate thickness, the load on the hydraulic cylinder is also increased, which lead to the error between actual position and the given position of hydraulic cylinder is increased. 3. Changes of PID parameters have a great influence on the displacement error of hydraulic cylinder in the process of site commissioning and position error decreases with the increase of PID parameters. But when the cylinder reaches the set position and return with a high-speed, the phenomenon of overshoot shock is going on in the hydraulic system and the entire piping system jitters badly. For safety, it is finally decided that the position error of hydraulic cylinder is increased and make sure the equipment runs as safely and steadily as possible. Built-in position sensor is used to detect the actual displacement of hydraulic cylinders, given displacement value of cylinders is used to minus the actual displacement values, the error is then multiplied by closed-loop feedback gain and calculation results is converted into a control voltage of the servo valve and then the servo valve core is driven, so the hydraulic cylinder can move to the given location. Four hydraulic cylinders are controlled by four independent servo valves respectively. Differences of displacements of each hydraulic cylinder must be controlled within the allowed error range of shear mechanism. According to reference, ${ }^{13}$ ) the maximum displacement error of hydraulic cylinder is $21.4 \mathrm{~mm}$. If the maximum permissible error is exceeded, the hydraulic system will be unstable. MTS position sensor is applied in this paper, precision of which is $0.01 \mathrm{~mm}$ and Rexroth 4WRTE35V1000L type high-response servo valve is applied. The maximum error of the actual detection is about $3 \mathrm{~mm}$ and the value is in the range of the maximum permissible error. Therefore, built-in sensor error value in this study does not affect the stability of the system.

By using Lyapunov method, hydraulic system stability is analyzed in theory, stable operating conditions of the system is obtained in this paper. As long as this condition is met, the action of rolling shear can be realized. At the same time, the Rexroth HNC controller is adopted, which achieve that the given displacement is better tracked by the actual displace- 


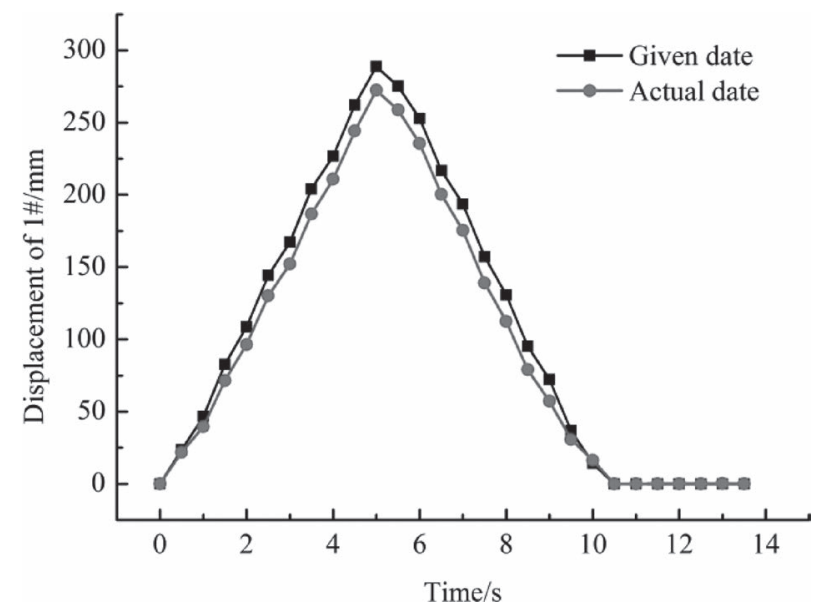

(a) The actual and given displacements of $1 \#$.

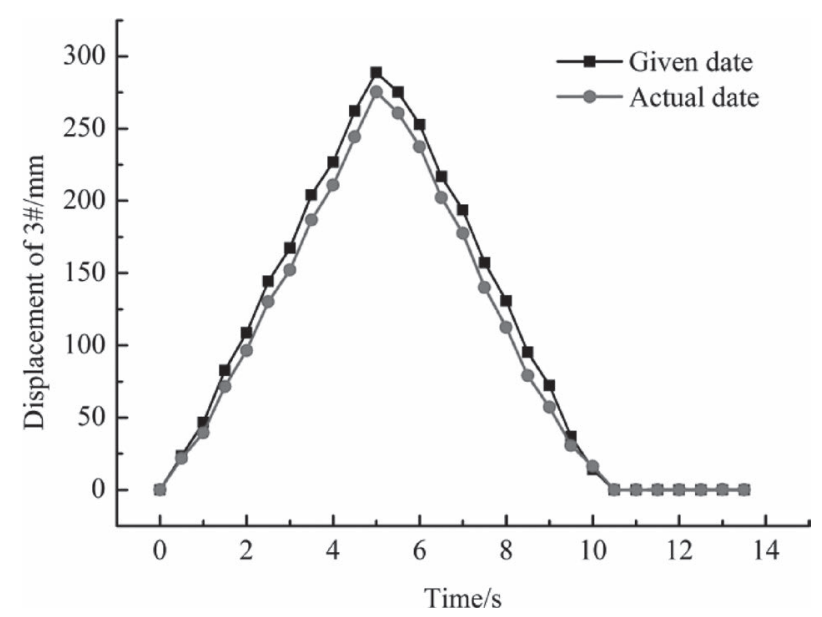

(c) The actual and given displacements of $3 \#$.

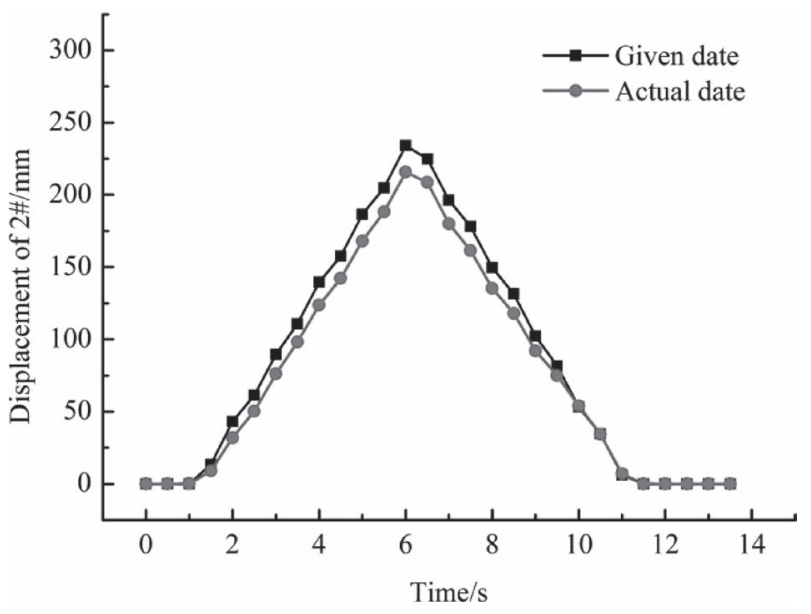

(b) The actual and given displacements of 2\#.

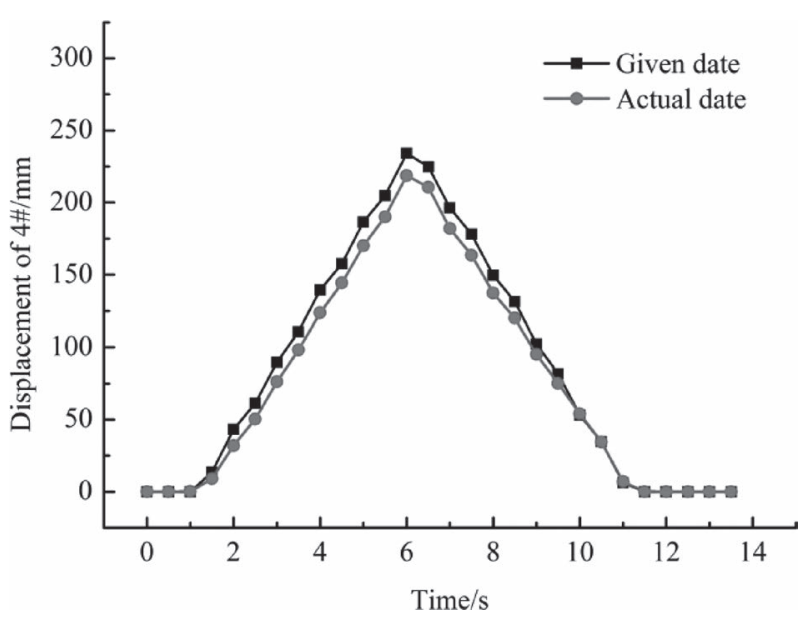

(d) The actual and given displacements of $4 \#$.

Fig. 3. The actual and given displacements of cylinders.

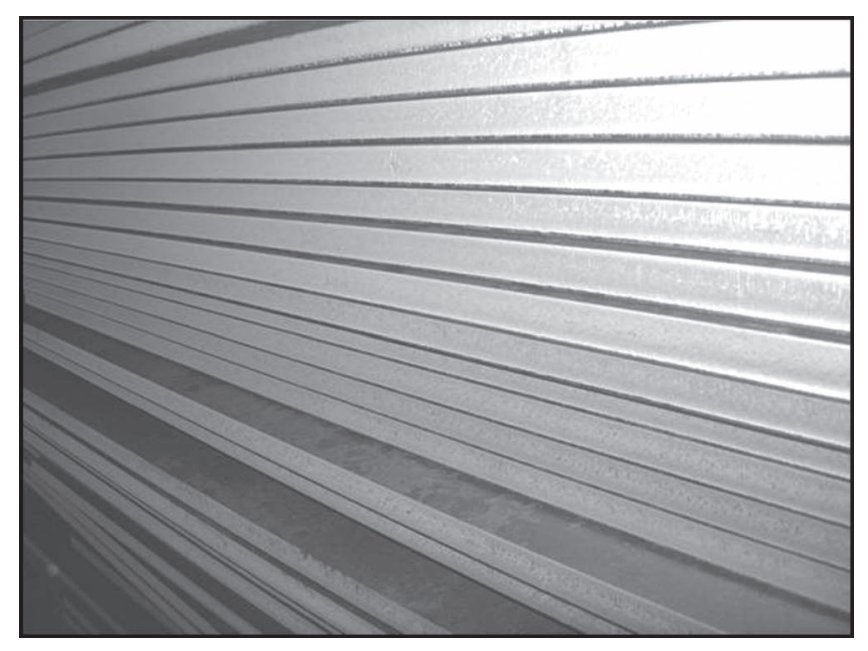

Fig. 4. The shear section of high quality.

ment of hydraulic cylinders. The tracking error of displacement reflects the robustness of the system and stability is also improved with robustness increased. ${ }^{14)}$ The shearing mechanism is directly controlled by the hydraulic system, which do shear motion of pure rolling. The stability of the system is necessary for achievement of rolling shear, which is conducive to achievement of the rolling shear movement and improvement of the quality of steel plate, as shown in

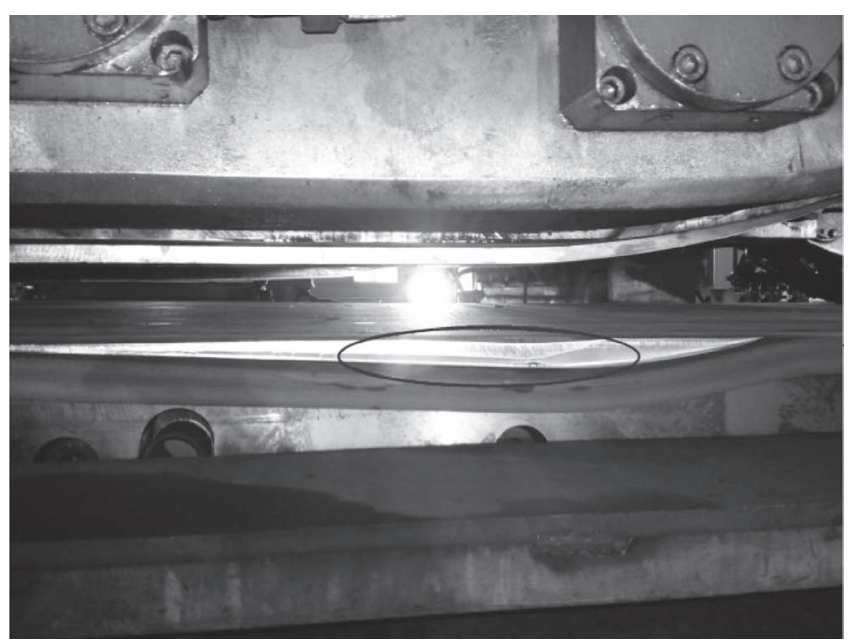

Fig. 5. Section profile tearing defects.

Fig. 4. Otherwise, the system is not stable, defects such as plate will be torn (as shown in Fig. 5).

The deviation of the positioning of the cylinders is considered in this paper. Instability shear phenomena of the new hydraulic bilateral rolling shear (as shown in Fig. 5) is occurred when the steel plate is sheared by upper blade and cylinder movement position is less than $26 \mathrm{~mm}$, which lead that shearing action of upper blade cannot be finished and 
position deviation of the four hydraulic cylinders is large. Therefore, when the system encounters an external load, it checks whether the motion displacement is greater than 26 $\mathrm{mm}$ for the bilateral rolling shear. If the displacement of the hydraulic cylinder is larger than $26 \mathrm{~mm}$, the system is stable and the shearing mechanism can finish the action of rolling shear. In addition, position closed-loop control accuracy of the hydraulic cylinder should be considered. The control precision and the rolling shear effect are both improved. Therefore, both theoretical and field data indicate that the hydraulic servo control system can satisfy the shearing track characteristics of the hydraulic rolling shear.

\section{Conclusions}

(1) In this study, by using unsymmetrical valve control unsymmetrical cylinder, the nonlinear equation of the state between force and cylinder flow is developed. Based on the mathematical model, displacement of the upper blade is greater than $26 \mathrm{~mm}$, the multi-cylinder hydraulic system of the shearing mechanism is stable.

(2) In this paper, by using the Liapunov's stability criterion, the method to verify stability of multi-cylinder system is deduced. Changing the parameters of the system, then the stability of the agency can be confirmed, which establishes an important theoretical foundation for analyzing stability of the system. This method of unsymmetrical valve control unsymmetrical cylinder is also suited to the system of works for null force.

(3) Stability of the multi-cylinder hydraulic system is evidenced according to the analysis of theory and experiment. The actual and given displacements in the field show that the method can satisfy the mode demand, and shows good feasibility and advantages.

\section{Acknowledgements}

This project is supported by National Natural Science Foundation of China (Grant No. 51505315, 51375325, 51404160), Provincial Fund for Young Scholars (2014011024-4), the initial funding of doctor research of Taiyuan University of Science and Technology (20122047).

\section{REFERENCES}

1) Z. B. Chu, Q. X. Huang and L. F. Ma: J. Sichuan Univ.: Eng. Sci. Ed., 43 (2011), 247.

2) Y. G. Bondar, Y. N. Belobrov and A. A. Kalashnikov: Metallurgist, 49 (2005), 456.

3) H. Y. Han, J. Wang and Q. X. Huang: Nonlinear Dyn., 70 (2012), 1199.

4) H. Y. Han, Q. X. Huang, J. Wang and J. Wang: Proc. Inst. Mech. Eng. B-J. Eng., Manuf., 227 (2013), 452.

5) D. Margolis and T. Shim: J. Dyn. Syst.-T. ASME, 125 (2003), 497.

6) Ch. Gentner, M. Sallaberger, Ch. Widmer, B. J. Bobach, H. Jaberg, J. Schiffer, F. Senn and M. Guggenberger: IOP: EES, 22 (2014).

7) J. Li, M. Tavakoli and Q. Huang: IEEE Trans., Control, Syst. Technol., 22 (2014), 2319

8) J. M. Vahid and M. Saleh: Nonlinear Dynam., 80 (2015), 591.

9) O. Keles and Y. Ercan: Control Eng. Pract., 10 (2002), 45.

10) E. E. Topç, I. Yüksel and Z. Kamis: Mechatronics, 16 (2006), 365.

11) C. X. Wang: Hydraulic Control System, China Machine Press, Beijing, (2011), 40.

12) H. Y. Han, J. Wang, J. Wang, L. F. Ma and Q. X. Huang: Proc. Inst. Eng. B-J. Eng. Manuf., 229 (2015), 1827.

13) Q. X. Huang, J. Li, H. Z. Li, H. Y. Han and L. D. Ma: ISIJ Int., 56 (2016), 288.

14) D. W. Clarke, C. Mohtadi and P. S. Tuffs: Automatica, 23 (1987), 137. 